\title{
EDITORIAL
}

\section{The effects of hypertension on aortic valve stenosis}

\author{
J Bermejo
}

Heart 2005;91:280-282. doi: 10.1136/hrt.2004.041749

\section{Hypertension not only can modify the exploratory findings of aortic stenosis, but may also interfere with the assessment of severity, and even have an impact on patient outcome}

Correspondence to: Javier Bermejo, $M D$, PhD, Laboratory of Échocardiography, Department of Cardiology, Hospital General Universitario Gregorio Marañón, Dr. Esquerdo 46. 28007 Madrid, Spain; javbermejo@jet.es ow values of systolic and pulse blood pressure have been classically considered hallmark signs of aortic valve stenosis (AS). However, hypertension has been shown to be independently associated with degenerative calcific aortic valve sclerosis and stenosis in elderly population based studies. ${ }^{12}$ Furthermore, in a recent series hypertension was found in one third of patients suffering symptomatic severe AS. ${ }^{3}$ In the absence of specific cohort studies, the nature of the association between AS and high blood pressure is not clear. Involved mechanisms may include a common aetiological pathway, a random coincidence of two highly prevalent diseases, or a true causal relation. Regarding the latter, essential hypertension may constitute a "classical" risk factor for developing AS, ${ }^{2}$ but also it seems that AS may cause systolic hypertension. ${ }^{4}$

\section{DIAGNOSTIC IMPLICATIONS}

Hypertension can modify the physical examination findings of AS, particularly in the elderly patient. $^{5}$ The characteristic exploratory findings of AS are all influenced by peripheral aortic impedance, wave reflections, poststenotic aortic dilatation, and vessel stiffness. Therefore, coexisting hypertension may result in a carotid pulse with a rapid upstroke and a normal amplitude, as well as in a diminished murmur or a near normal second cardiac sound. Because of these limitations, an echocardiographic examination should never be obviated if a systolic murmur is found, or if AS is suspected in a hypertensive subject. ${ }^{6}$

If AS coexists with hypertension, the valvar obstruction and the increased vascular resistance complementarily raise the systolic stress of the double loaded left ventricle. ${ }^{7}$ Moreover, these two sources of afterload interact following a characteristic behaviour. The intrinsic systolic load (that is, the valvar pressure gradient) is competitively modified by the extrinsic load (the systemic blood pressure) in an opposite and parallel fashion. ${ }^{7}$ This mechanism explains why vascular biomechanics may also modify the quantification of AS severity. However, the magnitude, clinical relevance, and diagnostic consequences of this vascular-valvar interaction are incompletely understood.
In this issue of Heart, Kadem and colleagues analyse the effects of systemic hypertension on AS indices in an animal model of supravalvar AS. ${ }^{8}$ Whether assessed invasively or by Doppler echocardiography, the authors found a consistent reduction of the transvalvar pressure gradient when arterial haemodynamics were manipulated by aortic clamping or phenylephrine infusion. In addition, the authors also report a close to $30 \%$ increase in effective valve area during banding of the descending aorta. The multivariate analysis failed to show any independent effect of systemic haemodynamic parameters on AS indices, and all the variation in the mean transvalvar pressure gradient was related to changes in transvalvar flow rate and valve area. Modification in the diameter of the proximal ascending aorta further accounted for the changes induced in the energy loss coefficient. Although showing a number of limitations (among the most important being an unrealistic modelling of valvar and of vascular haemodynamics) the study elegantly demonstrates how acute hypertension may induce a significant reduction in transvalvar flow rate, an increase in effective valve area, and consequently a reduction in the pressure gradient. ${ }^{8}$

\section{CHANGES IN AS SEVERITY}

Changes in AS severity indices induced by haemodynamic interventions can be readily explained by the Gorlin formula. Although alternative models of the pressure-flow relation in AS have been formulated, in vitro ${ }^{9}$ and in vivo $^{10}$ studies have clearly validated the physical principles of the Gorlin formula in AS. According to the Gorlin equation, for the pressure gradient to decrease, the transvalvar flow rate needs to decrease, the effective valve area must increase, or both. Thus, finding no independent association of the pressure gradient with arterial haemodynamic parameters such as aortic compliance, vascular resistance, or arterial elastance is not surprising, since their effect necessarily must be mediated by changes in flow rate and/or valve area.

Acting as an additive contributor to total afterload, a direct effect of blood pressure on transvalvar flow rate should be expected. Thus, the impact of blood pressure on the pressure gradient is straightforward. How arterial haemodynamics may modify valve area is less clear, and, unfortunately, the model of the study by Kadem and colleagues ${ }^{8}$ cannot be directly extrapolated to the valvar haemodynamics found in clinical AS. Flow may have a direct effect on Gorlin derived valve area, as well as on its noninvasive equivalent, the energy loss coefficient. Since these two indices depend on the amount of 
distal pressure recovered, they both may be influenced by changes of the diameter of the ascending aorta. ${ }^{11}$ In the clinical setting, AS severity is most frequently assessed by calculating the effective valve area using the continuity equation. ${ }^{6}$ Consistent evidence has demonstrated that continuity equation valve area also depends on flow rate, particularly in the presence of a reduced cardiac output. ${ }^{12}{ }^{13}$ Whether this effect is related to a flow mediated modification of the profile of the AS jet, of the contraction coefficient, ${ }^{14}$ or of the true orifice area, is a matter of debate. Irrespective of the mechanisms involved, effective valve area is far less flow dependent than the pressure gradient, and should be the index of choice for quantifying AS in the presence of abnormal flow rate or elevated blood pressure. ${ }^{6}$ Nevertheless, further studies using more realistic models of AS are needed to specifically address the effect of aortic pressure on valve area.

Arterial compliance and vascular resistance may modify Gorlin derived area values by an additional mechanism. Because the transvalvar pressure gradient is inverted during late ejection, we have shown that accounting only for valvar haemodynamics while the pressure gradient is positive may be misleading. ${ }^{15}$ Predictor analysis identified that a low arterial compliance and an increased systemic vascular resistance are direct contributors of a prolonged reverse gradient. Importantly, this source of error can be avoided using the incisura of the aortic pressure tracing to determine the end of the ejection period, instead of using the second left ventricular-aortic pressure crossover. ${ }^{15}$

\section{IMPACT ON PROGNOSIS}

Hypertension is a well established risk factor for cardiovascular events in the general population. In the absence of large scale cohort studies, the potential impact of coexistent hypertension on the outcome of AS patients is unknown. However, indirect evidence supports the proposal that hypertension may modify the symptomatic status (and hence the need for valve replacement) of AS patients. In an ambispective longitudinal study involving more than 300 patients, we have shown that left ventricular percentage stroke work loss is more useful than the pressure gradient and valve area to determine the outcome of unselected patients with AS. ${ }^{16}$ According to its definition formula, stroke work loss can be interpreted as a blood pressure normalisation of the pressure gradient. Thus, among patients with similar values of valve area and pressure gradient, those with lower blood pressure will develop AS related events sooner during follow up. Irrespective of valve area, higher values of systemic blood pressure seem to account for a less critical disease in terms of outcome.

\section{IMPLICATIONS FOR TREATMENT}

As previously discussed, a low arterial compliance and, most importantly, an increased vascular resistance, additively contribute to the increased systolic load caused by the outflow obstruction. In consequence, vascular resistance may be a major determinant of cardiac output when systolic stress is high due to ventricular dilatation, depressed contractility, and afterload mismatch. In this scenario, nitroprusside has been shown to induce a favourable effect on acute haemodynamics, even though the left ventricular pressure relief response is blunted due to a parallel increase in the transvalvar pressure gradient. ${ }^{17}$

It is far less clear whether a similar effect can be achieved by oral long term vasodilator treatment in asymptomatic patients with AS. Furthermore, the best drug for lowering blood pressure in the presence of an AS has not been established. A specific potential benefit has been suggested for inhibitors of the angiotensin converting enzyme (ACE), since ACE activity has been involved not only in an unfavourable left ventricular remodelling response, but also in the progression of valve degeneration. However, use of vasodilators has been classically considered contraindicated in AS, because of their potentially hazardous effect related to the risk of decreasing coronary perfusion pressure in this population. Although there are no empirical data to support it, this hypothesis definitely cannot be overlooked. Patients with AS are known to have a diminished coronary flow reserve, as do patients with ischaemic heart disease. It is remarkable that lowering diastolic pressure below $80 \mathrm{~mm} \mathrm{Hg}$ has been shown to increase the risk of myocardial infarction in ischaemic patients. ${ }^{18}$ Consequently, the particular "J shaped" curve of the blood pressure-risk relation needs to be specifically clarified for AS. Until the results of randomised placebo controlled studies become available, the role of these drugs should not be generalised.

In hypertensive patients with AS, arterial blood pressure may frequently revert to normal values after aortic valve replacement. ${ }^{4}$ If this would not be the case, antihypertensive medication should be initiated soon thereafter, because a raised blood pressure blunts the favourable regression of left ventricular hypertrophy obtained after valve replacement.

In conclusion, systemic hypertension is nowadays a frequent finding in patients with AS. A raised blood pressure may not only mask the exploratory findings of AS, but can also modify the haemodynamic indices used to assess the severity of the disease. These facts need to be considered for an adequate management of patients with AS.

Potential conflicts of interest: None to disclose.

\section{REFERENCES}

1 Stewart BF, Siscovick D, Lind BK, et al. Clinical factors associated with calcific aortic valve disease. Cardiovascular health study. J Am Coll Cardiol 1997;29:630-4.

2 Lindroos M, Kupari M, Heikkila J, et al. Prevalence of aortic valve abnormalities in the elderly: an echocardiographic study of a random population sample. J Am Coll Cardiol 1993;21:1220-5.

3 Antonini-Canterin F, Huang G, Cervesato E, et al. Symptomatic aortic stenosis: does systemic hypertension play an additional role? Hypertension 2003;41:1268-72.

4 le E, Mook W, Shapiro AP. Systolic hypertension in critical aortic stenosis and the effect of valve replacement. J Hum Hypertens 1996;10:65-7.

5 Das P, Pocock C, Chambers J. The patient with a systolic murmur: severe aortic stenosis may be missed during cardiovascular examination. QJM 2000;93:685-8.

6 Bonow RO, Carabello B, de Leon AC Jr, et al. Guidelines for the management of patients with valvar heart disease: executive summary. A report of the American College of Cardiology/American Heart Association task force on practice guidelines (committee on management of patients with valvular heart disease). Circulation 1998;98:1949-84.

7 Pasipoularides A. Clinical assessment of ventricular ejection dynamics with and without oufflow obstruction. J Am Coll Cardiol 1990;15:859-82.

8 Kadem L, Dusmenil JG, Rieu R, et al. Impact of systemic hypertension on the assessment of aortic stenosis. Heart 2005;91:354-61.

9 Voelker W, Reul H, Nienhaus G, et al. Comparison of valvular resistance, stroke work loss, and Gorlin valve area for quantification of aortic stenosis. An in vitro study in a pulsatile aortic flow model. Circulation 1995:91:1196-204

10 Bermejo J, Antoranz JC, Burwash IG, et al. In-vivo analysis of the instantaneous transvalvular pressure difference-flow relationship in aortic valve stenosis: implications of unsteady fluid-dynamics for the clinical assessment of disease severity. J Heart Valve Dis 2002; 1 1:557-66.

11 Garcia D, Dumesnil JG, Durand LG, et al. Discrepancies between catheter and Doppler estimates of valve effective orifice area can be predicted from the pressure recovery phenomenon: practical implications with regard to quantification of aortic stenosis severity. J Am Coll Cardiol 2003;41:435-42

12 Burwash IG, Thomas DD, Sadahiro M, et al. Dependence of Gorlin formula and continuity equation valve areas on transvalvular volume flow rate in valvular aortic stenosis. Circulation 1994;89:827-35.

13 Bermejo J, Garcia-Fernandez MA, Torrecilla EG, et al. Effects of dobutamine on Doppler echocardiographic indexes of aortic stenosis. J Am Coll Cardiol 1996;28:1206-13.

14 DeGroff CG, Shandas R, Valdes-Cruz L. Analysis of the effect of flow rate on the Doppler continuity equation for stenotic orifice area calculations: a numerical study. Circulation 1998;97:1597-605 
15 Bermejo J, Rojo-Álvarez JL, Antoranz JC, et al. Estimation of the end of ejection in aortic stenosis: an unreported source of error in the invasive assessment of severity. Circulation 2004; 110:1114-20.

16 Bermejo J, Odreman R, Feijoo J, et al. Clinical efficacy of Dopplerechocardiographic indices of aortic valve stenosis: a comparative test-based analysis of outcome. J Am Coll Cardiol 2003;41:142-51.
17 Khot UN, Novaro GM, Popovic ZB, et al. Nitroprusside in critically ill patients with left ventricular dysfunction and aortic stenosis. N Engl J Med 2003;348:1756-63

18 Bakris GL, Gaxiola E, Messerli FH, et al. Clinical outcomes in the diabetes cohort of the international verapamil SR-trandolapril study. Hypertension 2004:44:637-42

\section{IMAGES IN CARDIOLOGY}

\section{Late recognition of left ventricular non-compaction by cardiovascular magnetic resonance}

A

13 year old boy was investigated for recurrent exertional syncope. Chest $x$ ray showed a globular cardiac silhouette and 12 lead ECG suggested left ventricular hypertrophy with strain pattern. Transthoracic echocardiography (TTE) revealed basal septal hypertrophy with systolic anterior motion (SAM) of the mitral valve. A diagnosis of hypertrophic cardiomyopathy (HCM) was made and $\beta$ blockers were started. Twenty years later, the patient reported symptoms of gradually worsening dyspnoea. The ECG was unchanged and TTE revealed mild apical but no basal hypertrophy. Additionally, there was a reduced A wave on mitral pulsed wave Doppler and impaired left ventricular long axis function suggestive of a restrictive filling pattern. Repeat TTE three years later confirmed mild apical hypertrophy in the absence of basal hypertrophy or SAM. The patient underwent cardiovascular magnetic resonance (CMR). This demonstrated dilatation of all four cardiac chambers with globally impaired biventricular function and functional mitral regurgitation. The left ventricular apex was thinned with prominent trabeculations (upper panels A-D). There was late gadolinium enhancement of the epicardium and mid wall in the basal and mid septal and anterior walls in association with varying degrees of wall motion abnormality (lower panels A-D). These features suggested a diagnosis of dilated cardiomyopathy secondary to isolated left ventricular non-compaction (IVNC). IVNC is an increasingly recognised and important cause of heart failure and ventricular arrhythmias. This case with late presentation illustrates the complementary role of CMR with late gadolinium enhancement in the evaluation of cases of suspected apical HCM.

A Varghese N G Fisher

D J Pennell a.varghese@rbh.nthames.nhs.uk
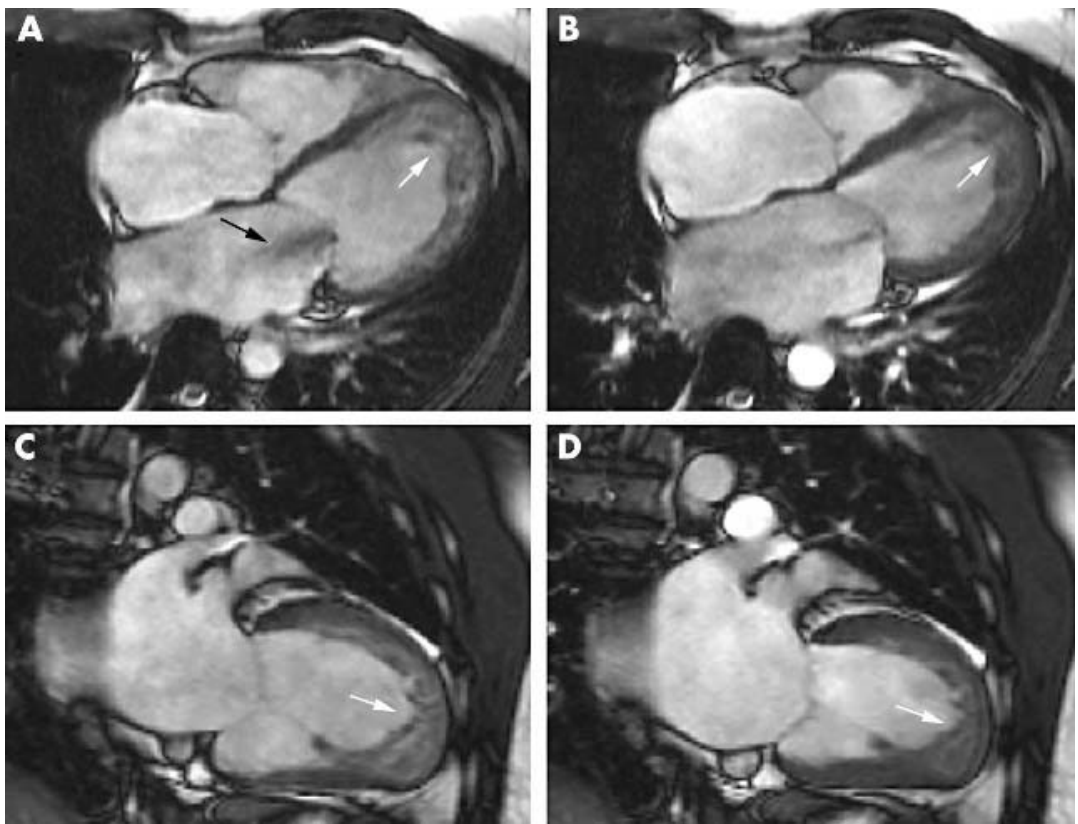

Panels A, B: four chamber view in (A) diastole and (B) systole. Panels C, D: ventricular long axis view in (C) diastole and (D) systole. There is heavy trabeculation of the apex of the left ventricle characteristic of IVNC (white arrows) with moderate mitral regurgitation (black arrow).
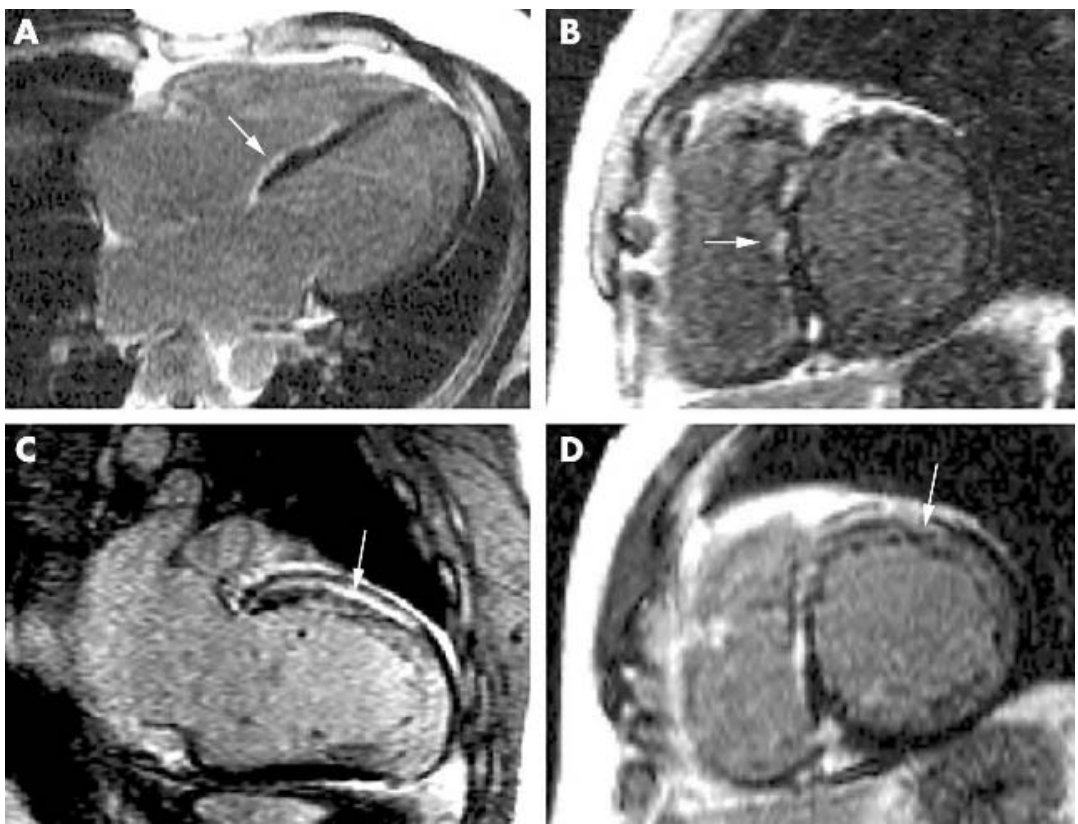

Panels A, B: four chamber (A) and basal short axis (B) views. Epicardial late gadolinium enhancement is present in the basal and mid septal wall (white arrows). Panels C, D: vertical long axis (C) and mid short axis (D) views. Mid wall late gadolinium enhancement is present in the anterior wall (white arrows). 